\title{
Three Cases of Canine Dermatomyositis-Like Disease
}

\author{
Camilo Romero', Genesis Garcia', Galia Sheinberg², Alberto Cordero³, Daniel Rodriguez ${ }^{4}$ \& Rafael Heredia'
}

\begin{abstract}
Background: Dermatomyositis is an idiopathic, inflammatory/immunemediated disease of the skin, muscles and blood vessels of hereditary nature and unclear pathogenesis. This familial disease has been described in certain breeds, especially collies and Shetland sheep dogs and is of rare occurrence in mongrel dogs. To describe and discuss three clinical cases of dermatomyositis-like disease and provide a brief review of the literature.

Cases: Three young mongrel dogs are included in this report. Case 1: Mandarino, a 4-year-old mongrel dog, having a history of skin lesions for at least a year. Showed an underweight patient, skin ulcers, crusts, alopecia, peri-ocular scarring causing severe lagophthalmia and a corneal ulcer. Muscle atrophy was most notable in the head and legs; the dog had difficulty and pain walking. Treatment was initialised with cephalexin $30 \mathrm{mg} / \mathrm{kg}$ BID, pentoxifylline $25 \mathrm{mg} / \mathrm{kg}$ BID, and prednisone $2.2 \mathrm{mg} / \mathrm{kg}$ SID. The patient was presented after two weeks for follow up; the anaemia and skin condition had improved, the weight had increased by $2 \mathrm{~kg}$, dysphagia and locomotor abnormalities were not present. Case 2: Milagros, a mongrel female dog approximately two years of age, rescued from a shelter. Physical examination showed facial alopecia, erythema and scarring of the periocular skin, crusting and scaling in alopecic areas, pinnae tip necrosis and crusting, ear alopecia, tail tip necrosis and crusting. Also present were distal limb alopecia, crusting and ulcers in areas of trauma in the hock and carpal surfaces; some nails presented onychorhexis and onychoschizia. The patient has been treated for 12 months with a good clinical outcome, with pentoxifylline, azathioprine $2.2 \mathrm{mg} / \mathrm{kg}$ EOD alternating with prednisone 1 $\mathrm{mg} / \mathrm{kg}$ EOD. Case 3: Chuchito, an 11-month-old male mongrel rescued dog had been previously hospitalised due to his skin condition. Physical examination showed depigmented and alopecic areas in the nasal planum, perioral and periocular areas, and inflammation of the palpebral tissues. Necrosis of the distal pinnae, alopecia and scales were evident, along with sloughing of scales and ulcers. Skin lesions were also present in the distal limbs, and alopecia, erythema and some crusting and scales in the carpal, tarsal and digital areas. Onychodystrophy was present in several digits. This study describes the physical examination and the clinical pathological findings, including skin scrapings, fungal cultures, and skin biopsies, in three dogs with dermatomyositis-like disease, as well as the clinical outcomes after slightly different treatment protocols were used. The biopsy results of two dogs showed ischaemic dermatopathy.

Discussion: The most common initial signs of the disease are erythema, desquamation and alopecia in the facial area, ears, distal limbs and pinnae in young puppies aged between two and six months of age, followed by pigmentary changes. Muscular lesions are uncommon; when present, they represent the most severe form of this disease. Dysphagia is a common sign and mega-oesophagus may be present. Patients with muscular disease can manifest difficulty walking, with a stiff high gait. The immune mediated pathogenesis of dermatomyositis can relate to triggering factors in some dogs, such as drugs, infections, paraneoplasms, or toxins. Other potential inducing stressors include oestrus, whelping and excessive solar exposure. Dermatomyositis-like or familiar dermatomyositis is diagnosed using clinical findings, histopathology of skin and muscle, and muscle physiology studies. Electromyography, breed predisposition and genetic background can be helpful in some cases. The clinical findings and response to the treatment of all three cases were compatible with dermatomyositis-like disease in mongrel dogs.
\end{abstract}

Keywords: dermatomyositis, dermatopathy, vascular disease, inflammatory myopathies, mongrel dog. 


\section{INTRODUCCTION}

Canine dermatomyositis is an uncommon skin disease that may affect muscles and vascular tissues $[5,8]$. Familial canine dermatomyositis (DM) is a hereditary idiopathic condition of the skin and muscles of young collies, Shetland, Beauceron, Belgian Tervuren and Portuguese water dogs; it has also been reported in the mongrel dog (familial canine dermatomyositis). A similar syndrome called dermatomyositis-like disease has also been also described in a Pembroke Welsh corgi, Lakeland terrier, Chow Chow, Jack Russell terrier, German shepherd dog, Kuvasz, and in mongrels $[4,6,10,16,17]$ and a Rottweiler [2].

Dermatomyositis is believed to be a vascular disease; in general, vascular diseases seem to be associated with an immunologic response that damages vascular components of the dermis and subcutaneous tissues. Cutaneous vasculitis may be associated with coexisting diseases, such as malignancies, drug reactions or connective tissue disorders [7]. In dogs, ischaemic dermatopathies include five subtypes: canine familial dermatomyositis; juvenile-onset ischaemic dermatopathy; focal post-rabies vaccination reaction; generalised vaccination-induced ischaemic dermatopathy; and adult-onset generalised ischaemic dermatopathy [13].

Pentoxifylline may not be sufficient to control the disease and the use of steroids might be necessary, such as prednisone and triamcinolone, or a combination of other drugs. Topical steroids, or tacrolimus $0.1 \%$ twice daily, might also help to control localised lesions.

\section{CASES}

Case 1. Mandarino, a 4-year-old mongrel dog was presented to the dermatology service, having a history of skin lesions for at least a year since it was rescued. He had aprevious diagnosis of ichthyosis, scabies and demodicosis, and was previously treated with different antibiotics for several weeks. He had also received weekly ivermectin subcutaneous injections (unknown dose and trademark) and chlorhexidine (unknow trademark) baths, with no clinical improvement.

No treatment was being administered at the time of presentation. The owner reported anorexia, dysphagia and a mild cough. Physical examination showed an underweight patient, skin ulcers, crusts, alopecia, scarring of the peri-oral muscles and skin causing exposure of the teeth and gums, peri-ocular scarring causing severe lagophthalmia and a corneal ulcer affecting the left eye (Figure 1A). Muscle atrophy was most notable in the head and legs; the dog had difficulty and pain walking. Also present were necrosis of the pinnae (Figure 1B), ulceration and alopecia of the tail and skin of the distal limbs (Figure 1C); the abdomen and prepuce was also affected (Figure 1D). There were crackling sounds on auscultation, no fever was detected, the patient was uncomfortable and in pain.

Skin scrapings were negative for mites, cytology showed evidence of secondary bacterial infection, a fungal culture was negative after 21 days. Thoracic radiographs were performed and an alveolar pattern in the ventral aspect of the right middle lung lobe was present, consistent with aspiration pneumonia. A complete blood count showed non-regenerative anaemia and neutrophilia with the presence of immature neutrophils; the blood chemistry revealed raised levels of total proteins and globulins. Biopsies were taken from muscle and different skin lesions; histopathology revealed no relevant changes in the muscle. Dermatopathology showed follicular atrophy, dermal fibrosis, interstitial infiltrate of mononuclear cells, hydropic degeneration of the epidermal basal layer and changes compatible with vascular disease.

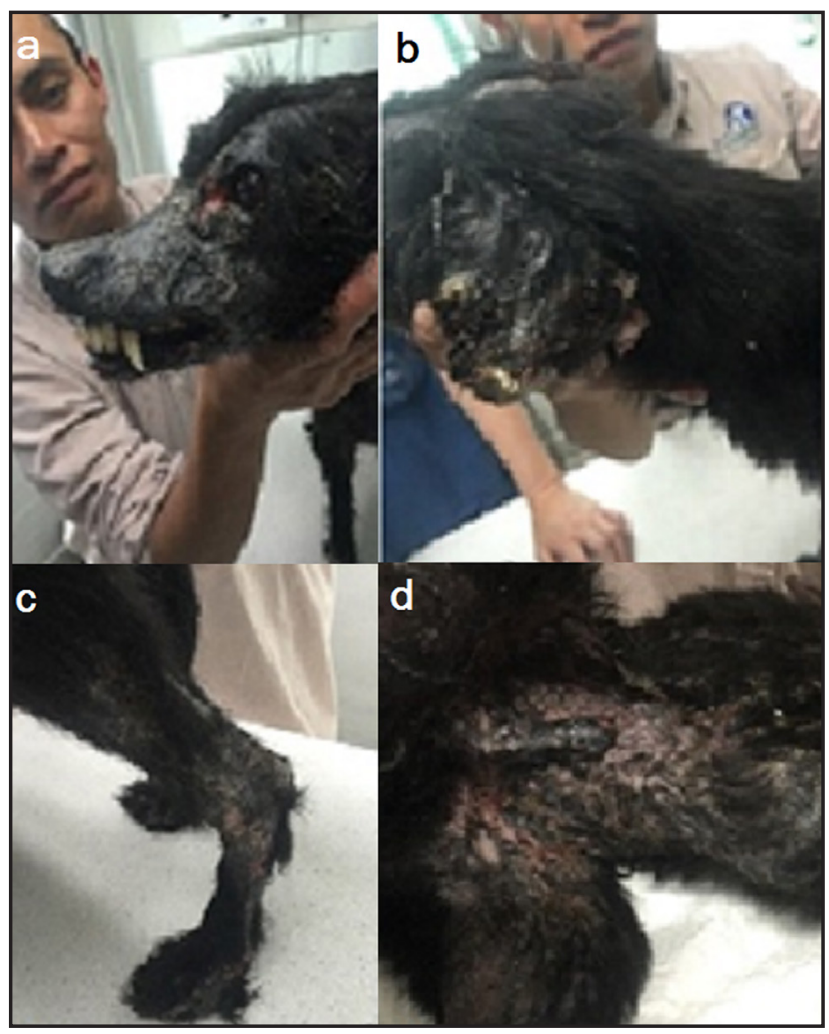

Figure 1. Muscle atrophy in the head and legs (A), necrosis of the pinnae (B), ulceration and alopecia (C), the abdomen and prepuce was also affected (D). 
Treatment was initialised with cephalexin $\left(\text { Keflex }^{\circledR}\right)^{1} 30 \mathrm{mg} / \mathrm{kg}$ BID, pentoxifylline $\left(\text { Elorgan }^{\circledR}\right)^{2}$ $25 \mathrm{mg} / \mathrm{kg} \mathrm{BID}$, and prednisone (Meticorten $\left.{ }^{\circledR}\right)^{3} 2.2 \mathrm{mg} /$ $\mathrm{kg}$ SID. Eye lubrication and medical therapy for the left eye ulcer was also instituted.

The patient was presented after two weeks for follow up; the anaemia and skin condition had improved, the weight had increased by $2 \mathrm{~kg}$, dysphagia and locomotor abnormalities were not present.

Ten weeks after initiating treatment, the patient started showing changes related to steroid therapy (marked polydipsia and polyuria); consequently, the prednisone was tapered slowly to $1 \mathrm{mg} / \mathrm{kg}$ but clinical signs continued and lesions started to reappear. Therapy with azathioprine $\left(\operatorname{Imuran}^{\circledR}\right)^{4}$ was instituted.

After eight months of treatment and disease remission, the patient was presented with neck and submandibular pain. A large painful subcutaneous mass was found; cytology showed poorly diferentiated cells with anisocytosis, polychromasia, macronucleosis and macrocariosis. No further tests were performed, and the dog was euthanised.

Case 2. Milagros, a mongrel female dog approximately two years of age, rescued from a shelter, was presented after treatment with prednisolone (Orapred $\left.^{\circledR}\right)^{5} 1 \mathrm{mg} / \mathrm{kg}$ for skin lesions prescribed by a general practitioner. The owner reported a slight improvement of the skin lesions at the beginning of the treatment three weeks before.

Physical examination showed facial alopecia, erythema and scarring of the periocular skin, crusting and scaling in alopecic areas, pinnae tip necrosis and crusting, ear alopecia (Figure 2A, B), tail tip necrosis and crusting. Also present were distal limb alopecia, crusting and ulcers in areas of trauma in the hock and carpal surfaces; some nails presented onychorhexis and onychoschizia.

Skin cytology was performed from the lesions, demonstrating non-degenerate neutrophils and nucleated keratinocytes. No microorganisms were found on cytology; a fungal culture was negative after 21 days. Skin biopsies were performed by the previous treating veterinarian showing inconclusive results, but the report was not made available to us. A CBC and blood chemistry showed normal parameters. The owners refused a second biopsy. Treatment with pentoxifylline $\left(\text { Elorgan }^{\circledR}\right)^{2}$ was initiated and the prednisone (Meticorten $\left.^{\circledR}\right)^{3}$ dose increased to $2 \mathrm{mg} / \mathrm{kg}$ SID; sun avoidance was recommended.

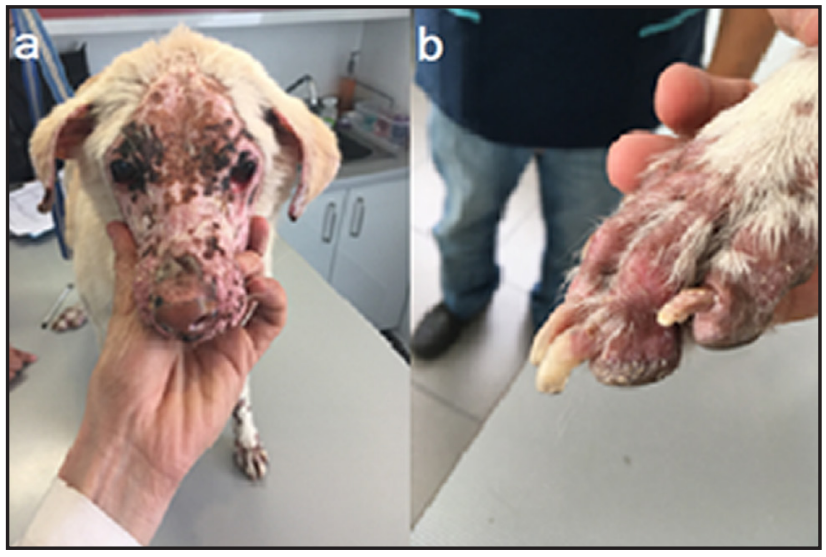

Figure 2. Facial alopecia, erythema and scarring of the periocular skin (A), crusting and scaling in alopecic areas (B), pinnae tip necrosis and crusting, ear alopecia.

After 30 days, a marked improvement was shown and so the therapy and prednisone was tapered. The lowest prednisone $\left(\text { Meticorten }^{\circledR}\right)^{3}$ dose without skin lesion recurrence was $1 \mathrm{mg} / \mathrm{kg} \mathrm{SID}$; azathioprine therapy was added to enable lowering the steroids needed for remission. The patient has been treated for 12 months with a good clinical outcome, with pentoxifylline $\left(\text { Elorgan }^{\circledR}\right)^{2}$, azathioprine $\left(\operatorname{Imuran}^{\circledR}\right)^{4} 2.2 \mathrm{mg} /$ $\mathrm{kg}$ EOD alternating with prednisone $\left(\text { Meticorten }^{\circledR}\right)^{3} 1$ $\mathrm{mg} / \mathrm{kg}$ EOD. Attempts to stop the steroid therapy were unsuccessful due to the lesions relapsing. The CBC and chemistry values are monitored every eight weeks. The patient is doing well at the writing of this article.

Case 3. Chuchito, an 11-month-old male mongrel rescued dog had been previously hospitalised due to his skin condition, and treated for mange with oral and injectable ivermectins (unknow dose and trademark) for three months, amitraz $\left(\text { Tactic }^{\circledR}\right)^{6}$ dips and oral cephalexin $\left(\operatorname{Keflex}^{\circledR}\right)^{1}$ for 6 weeks by the referring veterinarian. No improvement was observed during this period.

Physical examination showed depigmented and alopecic areas in the nasal planum, perioral and periocular areas, and inflammation of the palpebral tissues (Figure 3A, B). Necrosis of the distal pinnae, alopecia and scales were evident (Figure 3C), along with sloughing of scales and ulcers. Skin lesions were also present in the distal limbs, and alopecia, erythema and some crusting and scales in the carpal, tarsal and digital areas (Figure 3D). Onychodystrophy was present in several digits. Skin cytology was unremarkable, and the fungal cultures were negative after 21 days, skin scrapings were negative.

Orthopaedic radiographs of the hip and hind limbs were performed that revealed proximal femoral 


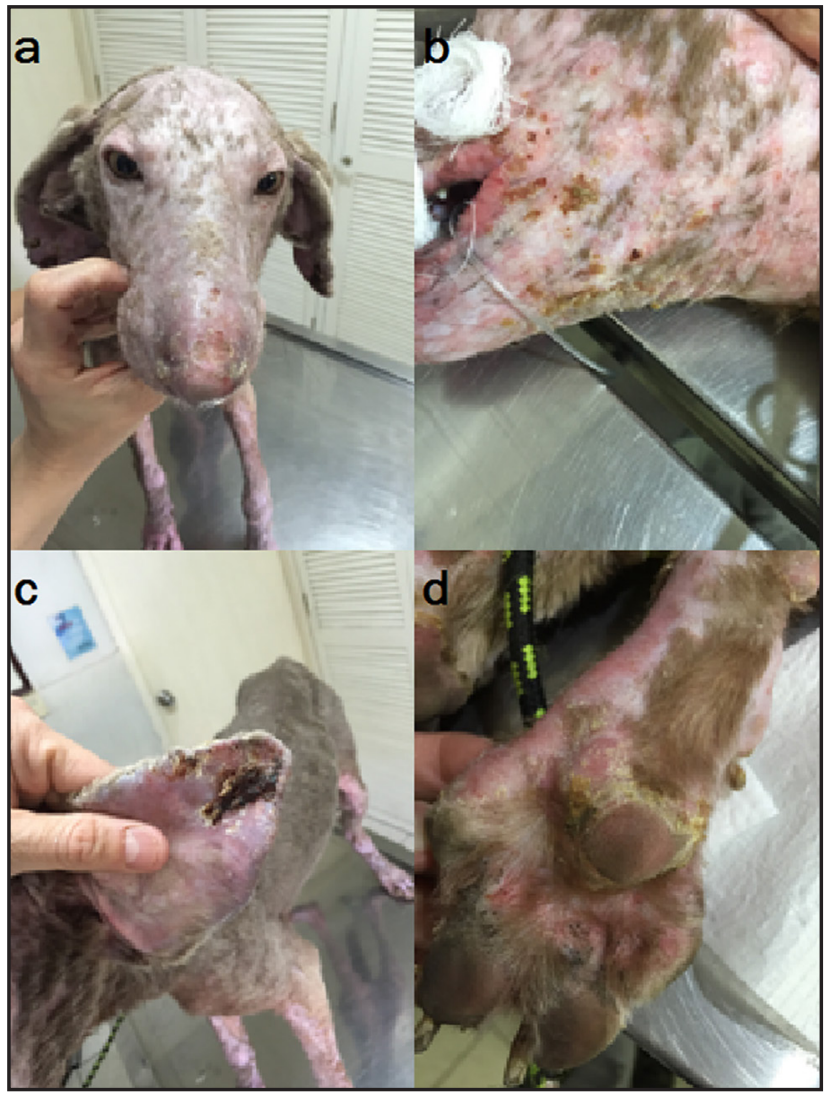

Figure 3. Depigmented and alopecic areas in the nasal planum, perioral and periocular areas, and inflammation of the palpebral tissues (A, B). Necrosis of the distal pinnae (C), skin lesions in the distal limbs, and alopecia, erythema and some crusting and scales in the carpal, tarsal and digital areas (D).

head and neck angular deformities. The right femur showed coxa-valga (femoral neck to proximal femoral shaft angle $>125$ degrees and a left femur showed coxa-vara (femoral neck to proximal femoral shaft angle $>125$ degrees) with secondary deformities of the proximal tibiae, more prominent on the right side. The right patella was slightly displaced laterally. No exact origin was determined but was believed to be either congenital or developmental. The abnormal proximal right hip angle probably affected the position of the right patella and was also likely to have contributed to the proximal deformities of the tibia.

The CBC, and blood chemistry were all within the normal range. Skin biopsies were taken from facial area in three different areas. The results are as follows: Superficial hair follicles are moderately hyperplastic and filled with compact keratin; very few hair shafts are present. Follicular growth activity is markedly reduced; most of the follicles are in telogen phase. Sebaceous gland lobules show variable atrophy and squamous metaplasia. Sweat glands are lined by flattened epithelium and contain inspissated secretory product. Dermal collagen bundles have a smudgy appearance, and there is mild dermal mucin deposition. The dermis has a mild, interstitial and periadnexal infiltrate comprising lymphocytes, pigmented macrophages and fewer neutrophils. No etiologic agents areobserved with routine histologic stains. Diagnosis: chronic ischaemic dermatopathy.

Treatment was initiated with prednisone (Meticorten $\left.^{\circledR}\right)^{3} 2.2 \mathrm{mg} / \mathrm{kg}$ day and pentoxifylline $\left(\text { Elorgan }^{\circledR}\right)^{2}$ $25 \mathrm{mg} / \mathrm{kg}$ BID. The patient developed a cough and diarrhea one week after treatment and so therapy was halted for 3 weeks during treatment for the respiratory condition. After the patient improved, treatment was re-initiated. Skin lesions improved partially but the owners noticed intense itching. Treatment options were discussed and changes were made; pentoxifylline $(\text { Elorgan } \AA)^{2}$ was continued at the same dosage, doxycycline $\left(\text { Vibramicina }^{\circledR}\right)^{7} 100 \mathrm{mg}$ BID, niacinamide $\left(\text { Niacin }^{\circledR}\right)^{8} 500 \mathrm{mg}$, and oclacitinib $\left(\text { Apoquel }^{\circledR}\right)^{9} 0.4 \mathrm{mg} /$ $\mathrm{kg}$ SID were initiated. After 8 weeks of therapy, the dog showed marked hair growth, nail quality improvement and no more active skin lesions. Solar exposure has not been limited and pigmentation is very noticeable in the alopecic areas.

\section{DISCUSSION}

Dermatomyositis may develop in any breed, including mongrel dogs; however, a genetic base has been demonstrated in certain breeds: Terveuren, Shetland sheepdog, Beauceron, and Portuguese water dog $[2,13]$, it is commonly manifested in young dogs in a very mild and self-limiting presentation, having spontaneous remissions after a few weeks. Disease progression may extend for months or years or throughout the dog's life $[1,11]$. In a study performed of 200 cases of inflammatory myopathy, the age ranged from 2 months to 14 years and no sex preference was found [4].

The most common initial signs of the disease are erythema, desquamation and alopecia in the facial area, ears, distal limbs and pinnae in young puppies aged between two and six months of age [5], followed by pigmentary changes [15]. Muscular lesions are uncommon; when present, they represent the most severe form of this disease. Dysphagia is a common sign and mega-oesophagus may be present. Patients with muscular disease can manifest difficulty walking, with a stiff high gait $[3,4,12]$. 
The immune mediated pathogenesis of dermatomyositis can relate to triggering factors in some dogs, such as drugs, infections, paraneoplasms, or toxins. Other potential inducing stressors include oestrus, whelping and excessive solar exposure [11].

Dermatomyositis-like or familiar dermatomyositis is diagnosed using clinical findings, histopathology of skin and muscle, and muscle physiology studies. Electromyography, breed predisposition and genetic background can be helpful in some cases [11,13].

The most interesting aspect of these cases is that all 3 dogs are mongrels or mixed breed, and all 3 are rescued. It is important to point out that, in central Mexico, there are no reported cases of leishmania which would be a differential diagnosis. As in many countries, economical resources are limited for diagnostic procedures in rescue groups, which was a problem we encountered treating these patients; electromyography was not available to our patients at the time of diagnosis. It could be argued that there are other potential diagnoses for these patients, such as vasculitis, vaccine-induced ischaemic dermatopathy, drug reactions, and other immune mediated diseases. All forms of ischaemic dermatopathy are characterised clinically by variable alopecia, erosions, ulcers, crusts, and hyperpigmention in a focal or multifocal distribution. Lesions can be localised to the dorsal interscapular region, as seen in post-rabies vaccination alopecia, or may multifocally involve the face, pinnae, foot pads, skin overlying boney prominences, and tip of the tail, as seen in DM and idiopathic ischaemic dermatopathy [14].

All patients showed very similar skin lesions with almost identical patterns (facial alopecia, crusting, ulceration and necrosis of pinnae, tail tip necrosis, distal limb skin lesions and nail involvement); in all three patients, solar radiation worsened the skin lesions, and two of the three cases had nail disease similar to that described in humans [9]. Histopathology aided the diagnosis in the two cases in which it was performed.

The first case, "Mandarino", was most severely affected and had the most dramatic improvement. The course of the disease was longer; there was muscular involvement, extensive scarring in the facial area and lagophthalmia due to eyelid scarring, the gums and teeth were also exposed because of lip scarring. This patient also developed aspiration pneumonia which we related to dysphagia. Once the skin lesions had gone into remission, the patient gained weight and blood parameters returned to normal. Unfortunately, this patient developed a malignancy that could have caused the immune mediated disease.

The last case, "Chuchito", was a young dog and the presentation was more typical. The cause of his limb deformity was not established, but a nutritional and developmental disorder was suspected. Interestingly, in this patient the treatment was not typical: steroids were not useful; he presented respiratory infections and gastrointestinal problems while on prednisone, so a doxycycline/niacinamide combination was used. This combination therapy has a immune-modulating effect that, combined with pentoxifylline, was very helpful in managing his skin lesions. However, itching (this has been reported in patients with dermatomyositis) was not controlled and oclacitinib was used successfully to inhibit it and make him more comfortable.

In conclusion, all three patients eventually responded well to treatments, but the first and third were more susceptible to steroid side effects; pentoxifylline was used in all three patients throughout the treatment process, and we believe that it was beneficial to all of them. Pentoxifylline is considered to be the best treatment for DM symtoms, but this depends on the severity of the disease. In these cases, we considered that this drug alone would not have been enough to manage the disease.

Azathioprine can be used safely in many patients with immune mediated diseases but monitoring of these patients is important. A calculated dose of azathioprine, using the body mass scale, is more effective and safer than a standard dose.

\section{MANUFACTURERS}

${ }^{1}$ Eli Lilly y Compañía de México, S. A. de C.V. Ciudad de México, México.

${ }^{2}$ Sanofi-Aventis S.A. Barcelona, Spain.

${ }^{3}$ Schering-Plough S.A. de C.V. Ciudad de México, México. ${ }^{4}$ Glaxo Smith Kline México S.A. de C.V. Ciudad de México, México.

${ }^{5}$ Sanofi-Aventis de México, S.A. de C.V. Ocoyoacac, México. ${ }^{6}$ Intervet México, S.A. de C.V. Santiago Tianguistenco, México.

${ }^{7}$ Pfizer S.A. de C.V. Toluca, México.

${ }^{8}$ Now Foods. Bloomingdale, IL, USA.

${ }^{9}$ Zoetis México, S. de R.L. de C.V. Ciudad de México, México.

Declaration of interest. The authors report no conflicts of interest. The authors alone are responsible for the content and writing of the paper. 


\section{REFERENCES}

1 Bensignor E. \& Guaguère E. 2007. La dermatomyosite canine familiale. Bulletin de l'Académie Vétérinaire de France. 160: 241-244.

2 Bresciani F., Zagnoli L., Fracassi F., Bianchi E., Cantile C., Abramo F. \& Pietra M. 2014. Dermatomyositis-like disease in a Rottweiler. Veterinary Dermatology. 25: 229-262.

3 Clark L.A., Credille K.M., Murphy K.E. \& Rees C.A. 2005. Brief communication: Linkage of dermatomyositis in the Shetland Sheep dog to chromosome 35. Veterinary Dermatology. 16: 392-394.

4 Evans J., Levesque D. \& Shelton G.D. 2004. Canine inflammatory myopathies: a clinic pathologic review of 200 cases. Journal of Veterinary Internal Medicine. 18: 679-691.

5 Ferguson E.A., Cerundolo R., Lloyd D.H., Rest J. \& Cappello R. 2000. Dermatomyositis in five Shetland sheepdogs in the United Kingdom. Veterinary Record. 146: 214-217.

6 Gross T.L., Ihrke P.J., Walder E.J. \& Affolter K.V. 2005. Vascular disease of the dermis. Interface diseases of the dermal-epidermal junction. In: Skin Diseases of the Dog and Cat. 2nd edn. Oxford: Wiley-Blackwell, pp.247-250.

7 Halliwell R.E.W. 2012. Autoimmune and immune-mediated dermatoses. In: Miller W.H., Miller W.H., Griffin C. \& Campbell K. (Eds). Muller and Kirk's Small Animal Dermatology. 7th edn. St. Louis: Elsevier, pp.432-500.

8 Hargis M.A., Prieur J.D., Haupt H.K., Evermann J.F. \& Ladiges W.C. 1986. Postmortem findings in four litters of dogs with familial canine dermatomyositis. The American Journal of Pathology. 123: 480-496.

9 Koler R.A. \& Montemarano A. 2001. Dermatomyositis. American Family Physician. 64: 1565-1572.

10 Miller W.H., Griffin C.E. \& Campbell K.L. 2012. Congenital and hereditary defects. Muller and Kirk's Small Animal Dermatology. 7th edn. St. Louis: Elsevier, pp.573-617.

11 Odaguiri J., Rossi C.N., Larsson Jr. C.E., Michalany N.S. \& Larsson C.E. 2012. Dermatomiosite canina familiarsimile: Relato de caso. Veterinária e Zootecnia. 19: 318-321.

12 Rees C.A. \& Boothe D.M. 2003. Therapeutic response to Pentoxifylline and its active metabolites in dogs with familial canine dermatomiositis. Journal of Veterinary Pharmacology an Therapeutics. 4: 234-241.

13 Röthig A., Rüfenacht S., Welle M.M. \& Thom N. 2015. Dermatomyositis in a family of Working Kelpies. Tierärztliche Praxis. 43: 331-336.

14 Vitale B.C., Gross L.T. \& Magro M.C. 1999. Vaccine-induced ischemic dermatopathy in the dog. Veterinary Dermatology. 10: 131-142.

15 Wahl J.M., Clark L.A., Skalli O., Ambrus A., Rees C.A., Mansell J.L. \& Murphy K.E. 2008. Analysis of gene transcript profiling and immunobiology in Shetland sheep dogs with dermatomyositis. Veterinary Dermatology. 19: 52-58.

16 White S.D., Shelton G.D., Sisson A., McPherron M., Rosychuk R.A.W. \& Olson P.J. 1992. Dermatomyositis in an adult Pembroke Welsh Corgi. Journal of the American Animal Hospital Association. 28: 398-401.

17 Yoon J.S., Minami T., Takizawa Y., Sekiguchi M., Yabuzoe A., Ide K., Nishifuji K. \& Iwasaki T. 2010. Two dogs with juvenile-onset skin diseases with involvement of extremities. Journal of Veterinary Medical Science. 72:1513-1516. 